Research Paper

\title{
Preoperative Thyroid Ultrasound Is Indicated in Patients Undergoing Par- athyroidectomy for Primary Hyperparathyroidism
}

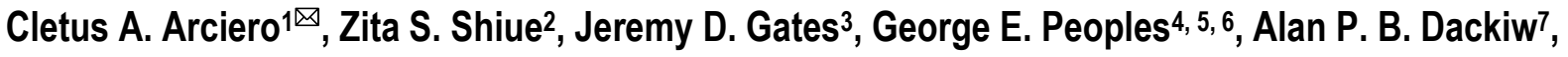 \\ Ralph P. Tufano ${ }^{7}$, Steven K. Libutti ${ }^{8,9}$, Martha A. Zeiger ${ }^{7}$, Alexander Stojadinovic ${ }^{5,6,9}$
}

1. Eisenhower Army Medical Center, Fort Gordon, GA, USA

2. University of Washington, Seattle WA, USA

3. Martin Army Community Hospital, Fort Benning, GA, USA

4. Brooke Army Medical Center, Fort Sam Houston, TX, USA

5. United States Military Cancer Institute, Washington, D.C. , USA

6. Uniformed Services University of the Health Sciences, Bethesda, MD, USA

7. Johns Hopkins University School of Medicine, Baltimore, MD, USA

8. Albert Einstein College of Medicine / Montefiore Medical Center, New York City, NY, USA

9. Walter Reed National Military Medical Center, Bethesda, MD, USA

$\triangle$ Corresponding author: Cletus Arciero, MD; Eisenhower Army Medical Center, Department of Surgery, Division of Surgical Oncology, Fort Gordon, GA. cletus.arciero@us.army.mil; Phone 706787 1142, FAX 7067871149.

(C) Ivyspring International Publisher. This is an open-access article distributed under the terms of the Creative Commons License (http://creativecommons.org/ licenses/by-nc-nd/3.0/). Reproduction is permitted for personal, noncommercial use, provided that the article is in whole, unmodified, and properly cited.

Received: 2011.10.27; Accepted: 2011.11.18; Published: 2011.11.25

\begin{abstract}
Background: Primary hyperaparathyroidism (pHPT) is often accompanied by underlying thyroid pathology that can confound preoperative parathyroid localization studies and complicate intra-operative decision making. The aim of this study was to examine the utility of preoperative thyroid ultrasonography (US) in patients prior to undergoing parathyroidectomy for pHPT.

Methods: An Institutional Review Board approved prospective study was undertaken from January 2005 through July 2008. All patients with pHPT meeting inclusion criteria $(n=94)$ underwent preoperative thyroid ultrasound in addition to standard ${ }^{99 m} \mathrm{Tc}$-sestamibi scintigraphy for parathyroid localization. Demographics, operative management and final pathology were examined in all cases.

Results: Fifty-four of the 94 patients $(57 \%)$ were noted to have a thyroid nodule on preoperative US, of which $30(56 \%)$ underwent further examination with fine needle aspiration biopsy. Alteration of the operative plan attributable to underlying thyroid pathology occurred in 16 patients $(17 \%)$, with patients undergoing either total thyroidectomy $(n=9)$ or thyroid lobectomy $(n=7)$. Thyroid cancer was noted in $33 \%$ of patients undergoing thyroid resection, and $6 \%$ of all patients with HPT.

Conclusions: The routine utilization of preoperative thyroid ultrasound in patients prior to undergoing parathyroid surgery for PHPT is indicated. The added information from this non-invasive modality facilitates timely management of co-incidental, and sometimes malignant, thyroid pathology.
\end{abstract}

Key words: hyperparathyroidism, thyroid, ultrasound, surgery, thyroid cancer.

\section{Introduction}

Primary hyperparathyroidism (pHPT) is a common endocrine disorder with an incidence of 0.4 to 1 per 1,000 individuals, with a peak during the 5 th and 6 th decades of life. ${ }^{1}$ Symptomatic patients, and those without symptoms that meet the guidelines for the 2002 NIH Consensus Development Panel for 
asymptomatic $\mathrm{pHPT}$, are candidates for parathyroid surgery. ${ }^{2}$ The incidence of thyroid cancer has progressively increased in the United States over the past decade. The treatment of thyroid carcinoma is surgical, and requires thyroid lobectomy / isthmusectomy, total thyroidectomy, or total thyroidectomy with central lymph node dissection (ATA Guidelines 2009), depending upon stage of disease. ${ }^{3}$ Concomitant pHPT in the setting of thyroid disease can present a surgical challenge for surgeons caring for these patients. Since Ogburn first described synchronous primary hyperparathyroidism and thyroid carcinoma more than fifty years ago, there have been multiple reports examining these co-existing endocrine disorders. ${ }^{4}$ Rates of co-existing primary hyperparathyroidism and thyroid pathology range from 20-67\%, with synchronous thyroid cancer occurring in 2 to $24 \%$. $4,5,6,7,8,9$

The standard surgical management of primary hyperparathyroidism has been bilateral neck exploration. This allowed the surgeon to examine all 4 parathyroid glands, but also allowed for a thorough examination of the thyroid gland. This approach often resulted in the identification and resection of concomitant thyroid disease. However, the modern management of primary hyperparathyroidism has moved towards minimally invasive approaches guided by nuclear medicine localization studies $(99 \mathrm{~m}$ Tc-sestamibi scintigraphy) and intra-operative parathyroid hormone (PTH) testing. This surgical approach allows for small incisions, lower morbidity, but less exposure of the thyroid gland, leading to concerns about missing co-existent, and significant thyroid pathology. We undertook a prospective, single-arm study examining all patients undergoing an operation for $\mathrm{pHPT}$ to determine the prevalence of concomitant thyroid disease detected by preoperative thyroid ultrasound.

\section{Material and Methods}

Specific Aim: To determine the prevalence of concomitant thyroid disease detected by preoperative thyroid ultrasound in patients undergoing surgery for pHPT.

Primary endpoint: The primary outcome variable in this study was prevalence of co-existent benign and malignant thyroid pathology.

Study population: Ninety-four of 110 patients screened for eligibility were enrolled in this study, conducted at two medical centers (Walter Reed Army Medical Center from January 2005 through June 2008; Johns Hopkins University Hospital from January 2008 through June 2008) under Institutional Review Board approval at the respective institutions and adherence to HIPAA compliance. Adult patients, age 18 years and older, with pHPT based upon standard diagnostic laboratory criteria, were eligible for inclusion. Patients unable to provide informed consent, those having previously undergone prior thyroid or parathyroid surgery, or those with known thyroid disease, patients with medullary thyroid carcinoma and/or multiple endocrine neoplasm syndrome, and those declining enrollment were excluded.

Study Design: This was a prospective observational cohort trial. All study patients initially underwent both laboratory screening and $99 \mathrm{~m}$ Tc-sestamibi scintigraphy. Operative planning was developed based on clinical findings and the results of the scintigraphy. Prior to surgical intervention, thyroid ultrasonography was performed and suspicious thyroid findings were further investigated with fine needle aspiration biopsy (FNAB) and/or thyroid resection. Any changes in the planned surgical procedure based on thyroid ultrasonography findings and/or FNA results were recorded.

Statistical Analysis: Descriptive statistics were used and study sample means are presented with standard deviations (mean $\pm \mathrm{SD}$ ).

\section{Results}

Clinical characteristics and Preoperative Evaluation: There were 110 patients evaluated and 94 patients enrolled: 71 females and 23 males, with a mean age of $56 \pm 13$ years (Table 1 ). Three patients reported a prior history of neck radiation, and a family history of thyroid cancer was identified in one patient who had a multi-nodular goiter (MNG). Preoperative laboratory evaluation confirmed hypercalcemia (mean: 11.0 $\pm 0.5 \mathrm{mg} / \mathrm{dl}$ ), elevated serum parathyroid hormone levels (mean: $117.9 \pm 50.7 \mathrm{pg} / \mathrm{ml}$ ) and elevated 24-hour urine calcium (mean: $367.5 \pm 180.3 \mathrm{mg} / 24$ hours) (Table 1). Each patient underwent preoperative $99 \mathrm{~m}$ Tc-sestamibi scintigraphy. Sestamibi identified parathyroid abnormalities in $86 \%(n=81)$ of patients suggesting a single parathyroid adenoma in $79 \%(n=74)$ and double parathyroid adenomas in $8 \%$ $(n=7)$. The remaining patients $(n=13,14 \%)$ had a non-localizing study, and underwent standard four gland exploration.

Preoperative Thyroid Ultrasound: Ultrasound examination revealed co-existent thyroid abnormalities in $57 \%(n=54)$. The abnormalities ranged from dominant nodules $<1 \mathrm{~cm}$ in 11 patients, dominant nodules $\geq 1 \mathrm{~cm}$ in 33 patients, and diffuse nodularity in the remaining 10 patients. Patients with thyroid pathology were slightly older (median age 60 versus 52) and more often female (M:F ratio 1:3.5 versus 1:2.6) $(\mathrm{p}=\mathrm{ns})$. The median size thyroid module size 
was $1.3 \mathrm{~cm}$ for all patients with thyroid pathology, and $1.5 \mathrm{~cm}$ for those patients that underwent FNAB $(n=31)$. Thyroid FNAB results included 3 indeterminate, 25 benign and 3 malignant/suspicious for malignancy (Table 2).

Operative Management: Alteration of the operative plan attributable to underlying thyroid pathology occurred in $17 \%(n=16)$ of patients. Of these 16 patients, in addition to a parathyroidectomy, nine underwent total thyroidectomy $(10 \%)$, and seven underwent thyroid lobectomy/isthmusectomy (6\%) (Figure 1).

Pathological Assessment: Evaluation of all resected parathyroid tissue confirmed that $96 \%(n=90)$ of patients had a solitary adenoma, 2\% ( $n=2)$ had double adenomas, $1 \%(n=1)$ had 4-gland hyperplasia, and $1 \%$ had a parathyroid carcinoma $(n=1)$. Among the 16 patients who underwent either thyroid lobectomy/isthmusectomy $(\mathrm{n}=7)$ or total thyroidectomy $(n=9), 5$ patients $(31 \%)$ had papillary thyroid carcinoma (PTC), 1 (6\%) patient had follicular thyroid carcinoma, 9 (55\%) had benign disease (MNG, follicular adenoma, Hurthle cell adenoma, adenomatoid nodule), and 1 (6\%) had an intra-thyroidal parathyroid adenoma (Figure 1). The prevalence of occult well-differentiated thyroid carcinoma in this study was $6 \%(6 / 94)$. There was no significant difference noted between patients with benign thyroid pathology and those with thyroid cancer in regards to patient age or size of lesion.

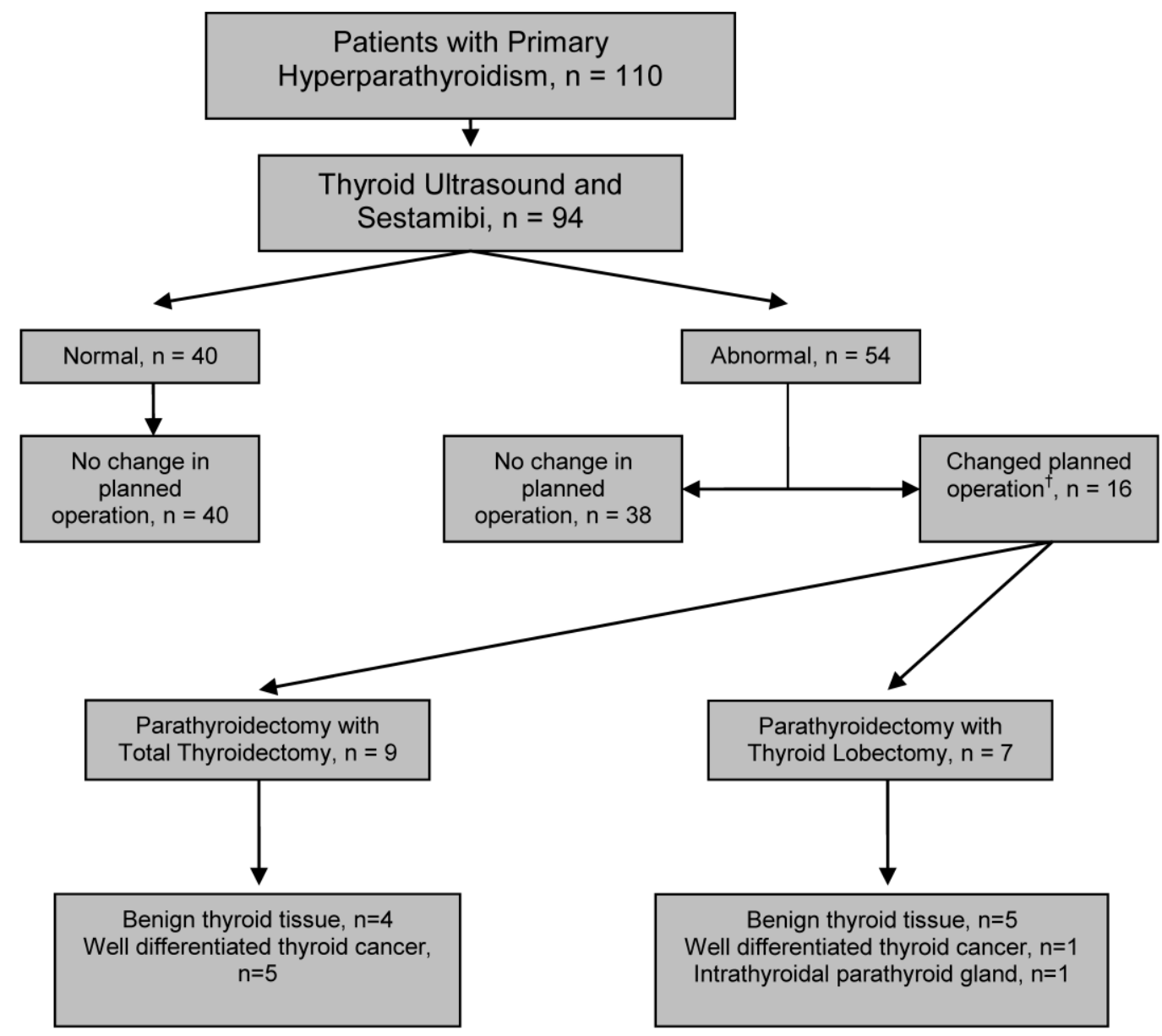

Thyroid US altered planned operation in 17\% (16/94) of patients.

Figure I: Evaluation of patients with PHPT by thyroid ultrasound and subsequent management based upon results. 
Table I: Patient demographics with pre-operative laboratory and radiographic results.

\begin{tabular}{ll}
\hline Demographics & Patients $(\mathbf{n}=\mathbf{9 4})$ \\
Age; Mean \pm SD (years) & $56.1 \pm 13.2$ \\
Gender (Female: Male) & $3.1: 1$ \\
Race (Caucasian: African American: Other) & $12.5: 2: 1$ \\
Prior neck radiation & 3 \\
Family history of thyroid cancer & 2 \\
Pre-Op Lab Test (n) & Mean \pm SD \\
Serum Calcium, mg/dl & $11.0 \pm 0.5$ \\
PTH, pg/ml & $117.9 \pm 50.7$ \\
Urine Calcium, mg/24hrs & $367.5 \pm 180.3(\mathrm{n}=44)$ \\
99m-Technetium Sestamibi Results & Patients $(\mathbf{n}=\mathbf{9 4})$ \\
Localized parathyroid abnormality & $81(86 \%)$ \\
Non-localized & $13(14 \%)$ \\
\hline
\end{tabular}

Table 2: Preoperative thyroid ultrasound results and resultant modifications in operative approach/procedure.

\begin{tabular}{ll}
\hline Preoperative Thyroid Ultrasound Results & Patients (n=94) \\
Thyroid Abnormalities Noted & $54(57 \%)$ \\
Suspicious Nodules & $31(33 \%)$ \\
Median Nodule Size & $1.3 \mathrm{~cm}$ \\
Fine Needle Aspirations Performed & Patients (n=31) \\
Pathology & \\
Indeterminate & $3(9.7 \%)$ \\
Benign & $25(80.6 \%)$ \\
Malignant; suspicious for malignancy & $3(9.7 \%)$ \\
Planned Operation (Pre-ultrasound) & Patients (n=94) \\
Minimally Invasive Parathyroidectomy & $87(92.5 \%)$ \\
Bilateral neck exploration & $7(7.5 \%)$ \\
Planned Operation (Post-Ultrasound) & Patients (n=94) \\
Minimally Invasive Parathyroidectomy & $72(77 \%)$ \\
Bilateral neck exploration & $22(23 \%)$ \\
Change in Operation (Addition of Thyroid & Patients (n=16) \\
Surgery) & 9 \\
Total Thyroidectomy & 7 \\
Thyroid Lobectomy / Isthmusectomy & 7 \\
\hline
\end{tabular}

\section{Discussion}

This study was undertaken to determine the prevalence of concomitant thyroid disease identified by preoperative thyroid US in patients undergoing surgical treatment for $\mathrm{pHPT}$. It was also designed to determine how often incidentally discovered thyroid pathology would alter the surgical management of these patients. Additionally, it provided the incidence of non-medullary thyroid cancer in patients undergoing surgical management of $\mathrm{pHPT}$.

Modern surgical management of $\mathrm{pHPT}$ has transitioned from the traditional bilateral neck explo- ration to minimally invasive parathyroidectomy (MIP). This minimally invasive surgical approach has allowed for outpatient surgical treatment of pHPT. The lack of preoperative thyroid imaging, however, can lead to missed and/or incompletely treated thyroid abnormalities. Previous studies have noted that in the performance of neck explorations, thyroid lesions $<1 \mathrm{~cm}$ in size are missed $94 \%$ of the time, and lesions that are $1-2 \mathrm{~cm}$ in size are missed $50 \%$ of the time. ${ }^{10}$ Milas and colleagues retrospectively examined 1,200 patients with pHPT who underwent bilateral neck exploration; 850 patients did not receive preoperative imaging and 350 patients underwent preoperative neck ultrasound revealing coexisting thyroid disease in $43 \%$. ${ }^{11}$ The lack of preoperative ultrasound led to a $30 \%$ thyroid resection rate while preoperative ultrasound resulted in $20 \%$ of patients undergoing FNAB and only $6 \%$ undergoing thyroid resection. In this current study, over $55 \%$ of patients with pHPT had thyroid abnormalities with $17 \%$ undergoing a thyroid resection. Identification of thyroid pathology leads to fewer thyroid resections. Often times, isolated thyroid nodules that have benign cytology on FNA can be safely followed with serial thyroid US thus avoiding the morbidity that can be associated with thyroidectomy, specifically recurrent laryngeal or superior laryngeal nerve injuries.

Historically, the prevalence of thyroid cancer in patients with $\mathrm{pHPT}$ ranged from 1 to $36 \%$. 8,12,13,14,15 More recent published studies report the coexistence of non-medullary thyroid cancer in $2-18 \%$ of patients operated on for pHPT. 58,16,17,18 Strichartz and Giuliano conducted a retrospective review of 308 patients who underwent an operation for hyperparathyroidism. ${ }^{8}$ Fifty-two $(17 \%)$ patients had grossly apparent thyroid abnormalities with histologically proven thyroid disease, and 11 (4\%) had differentiated thyroid cancer. Recently, in a retrospective review of 200 patients with pHPT, Morita, et al., noted that $51 \%$ of patients with $\mathrm{pHPT}$ had concomitant thyroid nodules and an overall $6 \%$ thyroid malignancy rate. ${ }^{5}$ Adler, et al., noted similar results with ultrasound detected thyroid abnormalities in $29 \%$ of patients with primary hyperaparathyroidism, although only $2 \%$ of patients were ultimately diagnosed with malignancy. ${ }^{19}$ In the 94 patients examined in this prospective study, it was noted that over $55 \%$ had coexistent thyroid pathology noted on preoperative thyroid US, which contributed to a change in surgical approach in $17 \%$. More importantly, $6 \%$ of patients underwent successful and timely treatment of well-differentiated thyroid cancer that may have otherwise been missed, presented at a more advanced stage, and/or required re-operative neck surgery. Although some might argue that occult 
well-differentiated thyroid cancer is of little clinical concern, is it important to note that the patients with occult well-differentiated thyroid cancer $(<1 \mathrm{~cm})$ have similar nodal and distant metastasis rates to patients with larger tumors $(>1 \mathrm{~cm}) .{ }^{20}$ Patients with identifiable thyroid abnormalities require diligent clinical investigation. Timely intervention for thyroid cancer avoids not only the risk and expense of neck re-exploration at a later time when the malignancy is clinically apparent but also the possibly more locally advanced disease when operation is delayed. Early diagnosis and timely treatment of thyroid cancer also avoids the medico-legal implications for a missed or delayed diagnosis.

Another concern of a missed or delayed diagnosis of a thyroid malignancy coincident with PHPT that should be noted is the requirement of re-operative surgery and the attendant risk of functionally limiting surgical morbidity. Although rates nerve injury rates of $\sim 1 \%$ are often quoted for thyroid and parathyroid resections, reoperative thyroid surgery has notable increases in morbidity rates with laryngeal nerve injury rates of $1-12 \%$ and hypocalcemia in $0.3-15 \%$ of patients. ${ }^{21,22,23,24,25,26}$ Thyroid surgery following previous parathyroid surgery is clearly not the optimal surgical situation. The combined operative management of simultaneous thyroid and parathyroid disease is preferred given its demonstrated safety, as it avoids the increased operative morbidity of a second neck exploration. ${ }^{14,15,16}$ This is particularly important in patients with unanticipated thyroid malignancy who can be treated safely at the time of initial operation for $\mathrm{pHPT}$.

This study reinforces what has previously been demonstrated in patients with pHPT; concomitant thyroid disease is not an infrequent occurrence. Preoperative thyroid ultrasound in patients with $\mathrm{pHPT}$ is useful adjunct to parathyroid scintigraphy and identifying concurrent thyroid disease. It has also been shown to be a cost-effective undertaking when utilized in conjunction with MIPS. ${ }^{13}$ With these points in mind, the debate about the nature and extent of treatment for isolated well-differentiated thyroid carcinoma is entertained in an entirely different context when considered in combination with $\mathrm{pHPT}$ requiring surgical treatment. In line with national endocrine disease treatment center practice patterns, our adoption of MIPS for pHPT attributable to a localizing adenoma precludes exploration of the entire neck and careful examination of the thyroid gland at time of operation. As such, timely diagnosis and treatment of the frequently encountered co-existing thyroid disease as shown in this study is advantageous, particularly considering the increased morbidity associated with a second neck exploration. Regardless of the surgical approach utilized, the inability to exclude coexistent thyroid pathology strictly on the basis of absence of palpable thyroid abnormality on clinical examination makes preoperative imaging of the thyroid gland with or without selective FNAB of detected thyroid abnormalities prior to parathyroidectomy justified. $6,16,27,28$

\section{Conflict of Interest}

There are no disclosures to report.

The views expressed in this manuscript are those of the authors and do not reflect the official policy of the Department of the Army, the Department of Defense or the United States Government.

Some of the contributing authors are military service members (or employee of the U.S. Government). This work was prepared as part of our official duties. Title 17 U.S.C. 105 provides the "Copyright protection under this title is not available for any work of the United States Government." Title 17 U.S.C. 101 defines a U.S. Government work as a work prepared by a military service member or employee of the U.S. Government as part of that person's official duties.

We certify that all individuals who qualify as authors have been listed; each has participated in one or more of the following areas: conception and design of this work, the analysis of data, the writing of the document. All contributing authors approve of the submission of this version of the manuscript and assert that the document represents valid work. If information derived from another source was used in this manuscript, we obtained all necessary approvals to use it and made appropriate acknowledgements in the document. All contributing authors take public responsibility for this work.

\section{References}

1. Silverberg SJ, Bilezikian JP. Hyperparathyroidism. In: Becker KL, editor. Principles and practice of endocrinology and metabolism, 3rd edition. Philadelphia: JB Lippincott. 2001: 564-573.

2. Bilezikian JP, Potts JT, El-Hajj Fuleihan G. Summary statement from a workshop on asymptomatic primary hyperparathyroidism: a perspective for the 21st century. J Clin Endocrinol Metab 2002;87:5353-5361.

3. Cooper DS, Doherty GM, Haugen BR, Kloos RT, Lee SL, Mandel SJ, Mazzaferri EL, McIver B, Pancini F, Schlumberger M, Sherman SI, Steward DL, Tuttle RM. Revised American Thyroid Association management guidelines for patients with thyroid nodules and differentiated thyroid cancer. Thyroid 2009;19(11):1-48.

4. Ogburn PL, Black BM. Primary hyperparathyroidism and papillary adenocarcinoma of the thyroid; report of four cases. Proc Staff Meet Mayo Clin 1956;31(10):295-298. 
5. Morita SY, Somervell H, Umbricht CB, Dackiw AP, Zeiger MA. Evaluation for concomitant thyroid nodules and primary hyperparathyroidism in patients undergoing parathyroidectomy or thyroidectomy. Surgery 2008;144:862-867.

6. Krause UC, Friedric JH, Olbricht T. Association of primary hyperparathyroidism and non-medullary thyroid cancer. European Journal of Surgery 1996;162:685-689.

7. Kosem M, Algun E, Kotan C. Coexistent thyroid pathologies and high rate of papillary cancer in patients with primary hyperparathyroidism: Controversies about minimal invasive parathyroid surgery. Acta Chir Belg 2004;104:568-571.

8. Strichartz SD, Giuliano AE. The operative management of coexisting thyroid and parathyroid disease. Arch Surg 1990;125(10):1327-1331.

9. Erbil Y, Salmasliolu A, Kabul E, Isever H, Tunaci M, Adalet I, Bozbora A, Ozarmaan S. Use of preoperative parathyroid fine-needle aspiration and parathormone assay in the primary hyperparathyroidism with concomitant thyroid nodules. Am J Surg 2007;193(6):665-671.

10. Brander A, Viikinkoski P, Tuuhea J, Voutilainen L, Kivisaari L. Clinical versus ultrasound examination of the thyroid gland in common clinical practice. J Clin Ultrasound 1992;20(1):37-42.

11. Milas M, Mensah A, Alghoul M, Berber E, Stephen A, Siperstein A, Weber CJ. The impact of office neck ultrasound on reducing unnecessary thyroid surgery in patients undergoing parathyroidectomy. Thyroid 2005;15(9):1055-1059.

12. Lever E, Refetoff S, Straus FH 2nd, Nguyen M, Kaplan EL. Coexisting thyroid and parathyroid disease - are they related? Surgery 1983;94(6):893-900.

13. Hollenbeak CS, Lendel I, Beus KS. The cost of screening for synchronous thyroid disease in patients presenting with primary hyperparathyroidism. Arch Otolaryngol Head Neck Surg 2007;133(10):1013-1021.

14. van der Schaar $H$ \& Mulder $H$. Lesions of the thyroid gland in patients with primary hyperparathyroidism. Surgery, Gynecology and Obstetrics 1985;160:407-408.

15. Kairaluoma MV. Simultaneous thyroid operation in patients undergoing initial neck exploration for primary hyperparathyroidism. Ann Chir Gynaecol 1994;83(1):30-34.

16. Regal M, Paramo C, Luna Cano R. Coexistence of primary hyperparathyroidism and thyroid disease. Journal of Endocrinological Investigation 1999;22:191-197.

17. Leitha T, Staudenherz A. Concomitant hyperparathyroidism and non-medullary thyroid cancer, with a review of the literature. Clinical Nuclear Medicine 2003;28(2):113-117.

18. Masatsugu T, Yamashita H, Noguchi S. Thyroid Evaluation in Patients with Primary Hyperparathyroidism. Endocrine Journal 2005;52(2):177-182.

19. Adler JT, Chen H, Schaefer S, Sipple RS. Does routine use of ultrasound result in additional thyroid procedures in patients with primary hyperparathyroidism? J Am Coll Surg; 2010;211:536-539.

20. Furlan JC, Bedard Y, Rosen IB. Biologic basis for the treatment of microscopic, occult well-differentiated thyroid cancer. Surgery 2001;130(6):1050-1054.

21. Tisell L, Hansson G, Jansson S, et al.. Reoperation in the treatment of asymptomatic metastasizing medullary thyroid carcinoma. Surgery 1986;99:60-6.

22. Goretzki P, Simon D, Frilling A, et al.. Surgical reintervention for differentiated thyroid cancer. Br J Surg 1993;80:1009-12.

23. Patou CA, Norton JA, Brennan MF. Hypocalcemia following thyroid surgery: incidence and prediction of outcome. World J Surg 1998;22:718-24.

24. Shaha AR, Jaffe BM. Parathyroid preservation during thyroid surgery. Am J Otol 1998;109:568-74.
25. Wingert DJ, Friesen SR, Illiopoulos JI, et al.. Post-thyroidectomy hypocalcemia: incidence and risk factors. Am J Surg 1986;152:606-10.

26. Levin KE, Clark AH, Duh QY, et al.. Reoperative thyroid surgery. Surgery 1992;111:604-7.

27. Sidhu S, Campbell P. Thyroid pathology associated with primary hyperparathyroidism. Australia and New Zealand Journal of Surgery 2000;70:285-287.

28. Regal M, Paramo C, Luna Cano R. Coexistence of primary hyperparathyroidism and thyroid disease. J Endocrinol Invest 1999;22:191-197. 